\title{
Transmuted Exponentiated Modified Weibull Distribution
}

\author{
S.K. Ashour* and M.A. Eltehiwy \\ Institute of Statistical Studies \& Research, Cairo University, Egypt \\ *Corresponding author E-mail: ashoursamir@hotmail.com
}

\begin{abstract}
A generalization of the exponentiated modified Weibull distribution so-called the transmuted exponentiated modified Weibull distribution is proposed and studied. Various structural properties including explicit expressions for the moments, quantiles, and moment generating function of the new distribution are derived. The estimation of the model parameters is performed by least square method. We hope that the new distribution proposed here will serve as an alternative model to the other models which are available in the literature for modeling positive real data in many areas.
\end{abstract}

Keywords: Exponentiated modified Weibull distribution, least square estimation, moment generating function, order statistics, reliability functions.

\section{Introduction}

The quality of the procedures used in a statistical analysis depends heavily on the assumed probability model or distributions. Because of this, considerable effort has been expended in the development of large classes of standard probability distributions along with revelent statistical methodologies. In fact, the statistics literature is filled with hundreds of continuous univariate distributions. However, in recent years, applications from the environmental, financial, biomedical sciences, engineering among others, have further shown that data sets following the classical distributions are more often the exception rather than the reality. Since there is a clear need for extended forms of these distributions a significant progress has been made toward the generalization of some well-known distributions and their successful application to problems in areas such as engineering, finance, economics and biomedical sciences, among others.

In this article we use transmutation map approach suggested by Shaw and Buckley [13] to define a new model which generalizes the Exponentiated modified Weibull model and extends recent development on transmuted modified Weibull distribution by Khan and King [7] .We will call the generalized distribution as the transmuted Exponentiated modified Weibull (TEMWD) distribution. According to the Quadratic Rank Transmutation Map, (QRTM), approach the cumulative distribution function (cdf) satisfy the relationship

$F_{2}(x)=(1+\lambda) F_{1}(x)-\lambda F_{1}(x)^{2}$

which on differentiation yields,

$f_{2}(x)=f_{1}(x)\left[1+\lambda-2 \lambda F_{1}(x)\right]$

where $f_{1}(x)$ and $f_{2}(x)$ are the corresponding probability density function (pdf) associated with $F_{1}(x)$ and $F_{2}(x)$ respectively and $-1 \leq \lambda \leq 1$. Extensive information about the quadratic rank transmutation map is given in Shaw and Buckley [13]. We will use the above formulation for a pair of distributions $F(x)$ and $G(x)$ where $G(x)$ is a submodel of $F(x)$. therefore, a random variable $X$ is said to have a transmuted probability distribution with cdf $F(x)$ if

$F(x)=(1+\lambda) G(x)-\lambda G(x)^{2}, \quad|\lambda| \leq 1$

where $G(x)$ is the cdf of the base distribution. Observe that at $\lambda=0$ we have the distribution of the base random variable. Aryal and Tsokos $[1,2]$ studied the transmuted extreme distributions. The authors provided the mathematical characterization of transmuted Gumbel and transmuted Weibull distributions and their applications to analyze real data sets. Aryal [3] studied the transmuted log logistic distribution and discussed some properties of this family. In the present study we will provide mathematical formulation of the transmuted exponentiated modified Weibull (TEMWD) distribution and some of its properties. 


\section{Transmuted exponentiated modified Weibull distribution}

Elbatal [4] generalized the modified Weibull distribution by powering a positive real number $(\alpha)$ to the cumulative distribution function (cdf). This new family of distributions called exponentiated modified Weibull distribution. The new distribution due to its flexibility in accommodating all the forms of the hazard rate function can be used in a variety of problems for modeling random lifetimes. Another important characteristic of the distribution is that it contains, as special cases, the Weibull, exponentiated exponential (Gupta and Kundu[5]), exponentiated Weibull distribution (Mudholkar et al.[11,12]), generalized Rayleigh (Kundu and Rakab[8]), modified Weibull distribution (Lai et al.[9]) and some other distributions.

A random variable $X$ is said to have a exponentiated modified Weibull distribution with parameters $\alpha, \beta, \theta$ and $\gamma>0$ if its probabability density function (pdf) is given by

$\mathrm{g}(\mathrm{x})=\alpha\left(\theta+\gamma \beta x^{\beta-1}\right) e^{-\left(\theta x+\gamma x^{\beta}\right)}\left[1-e^{-\left(\theta x+\gamma x^{\beta}\right)}\right]^{\alpha-1}, \theta, \alpha, \beta$ and $\gamma>0$

and the cdf of $X$ is given by

$G(x)=\left[1-e^{-\left(\theta x+\gamma x^{\beta}\right)}\right]^{\alpha} . x>0, \quad \theta, \alpha, \beta$ and $\gamma>0$

Now using (3) and (5) we have the cdf of a transmuted exponentiated modified Weibull distribution as follows:

$F(x)=\left[1-e^{-\left(\theta x+\gamma x^{\beta}\right)}\right]^{\alpha}\left((1+\lambda)-\lambda\left[1-e^{-\left(\theta x+\gamma x^{\beta}\right)}\right]^{\alpha}\right)$

Hence, the pdf of the transmuted exponentiated modified Weibull distribution with parameters $\theta, \alpha, \beta, \gamma$ and $\lambda$ is

$f(x)=\alpha\left(\theta+\gamma \beta x^{\beta-1}\right) e^{-\left(\theta x+\gamma x^{\beta}\right)}\left[1-e^{-\left(\theta x+\gamma x^{\beta}\right)}\right]^{\alpha-1}\left(1+\lambda-2 \lambda\left[1-e^{-\left(\theta x+\gamma x^{\beta}\right)}\right]^{\alpha}\right)$

where $\gamma, \beta$ and $\alpha$ are the shape parameters representing the different patterns of the transmuted exponentiated modified Weibull distribution and are positive, $\theta$ is a scale parameter representing the characteristic life and is also positive, $\lambda$ is the transmuted paramter. The restrictions in equation (7) on values of $\theta, \alpha, \beta, \gamma$ and $\lambda$ are always the same.

The transmuted exponentiated modified Weibull distribution is very flexible model that approaches to different distributions when its parameters are changed. We present special cases of the transmuted exponentiated modified Weibull distribution (TEMWD) as follows:

Exponentiated modified Weibull distribution. for $\lambda=0$, the distribution function (6) becomes

$F(x)=\left[1-e^{-\left(\theta x+\gamma x^{\beta}\right)}\right]^{\alpha}$

which is the distribution function of the exponentiated modified Weibull distribution.

Modified Weibull distribution. For $\lambda=0, \alpha=1$, the distribution function (6) becomes

$F(x)=1-e^{-\left(\theta x+\gamma x^{\beta}\right)}$

which is the distribution function of the modified Weibull distribution.

Transmuted modified Weibull distribution. For $\alpha=1$, the distribution function (6) becomes

$F(x)=\left(1-e^{-\left(\theta x+\gamma x^{\beta}\right)}\right)\left(1+\lambda e^{-\left(\theta x+\gamma x^{\beta}\right)}\right)$

Exponentiated Weibull distribution. For $\lambda, \theta=0$, the distribution function (6) becomes 
$F(x)=\left[1-e^{-\left(\gamma x^{\beta}\right)}\right]^{\alpha}$

Transmuted exponentiated Weibull distribution. For $\theta=0$, the distribution function (6) becomes

$F(x)=\left[1-e^{-\left(\gamma x^{\beta}\right)}\right]^{\alpha}\left((1+\lambda)-\lambda\left[1-e^{-\left(\gamma x^{\beta}\right)}\right]^{\alpha}\right)$

Weibull distribution. For $\lambda, \theta=0, \alpha=1$, the distribution function (6) becomes

$F(x)=1-e^{-\left(\gamma x^{\beta}\right)}$

Transmuted Weibull distribution. For $\theta=0, \alpha=1$, the distribution function (6) becomes

$F(x)=\left[1-e^{-\left(\gamma x^{\beta}\right)}\right]\left[1+\lambda e^{-\left(\gamma x^{\beta}\right)}\right]$

Exponentiated exponential distribution. For $\lambda, \theta=0, \beta=1$, the distribution function (6) becomes

$F(x)=\left[1-e^{-(\gamma x)}\right]^{\alpha}$

which is the distribution function of the exponentiated exponential distribution introduced by Gupta and Kundu [5].

Transmuted exponentiated exponential distribution. For $\theta=0, \beta=1$, the distribution function (6) becomes

$F(x)=\left[1-e^{-(\gamma x)}\right]^{\alpha}\left((1+\lambda)-\lambda\left[1-e^{-(\gamma x)}\right]^{\alpha}\right)$

Exponential distribution. For $\lambda, \theta=0, \alpha, \beta=1$, the distribution function (6) becomes

$F(x)=1-e^{-(\gamma x)}$

Transmuted Exponential distribution. For $\theta=0, \alpha, \beta=1$, the distribution function (6) becomes

$F(x)=\left[1-e^{-(\gamma x)}\right]\left[1+\lambda e^{-(\gamma x)}\right]$

Generalized linear failure rate distribution. For $\lambda=0, \beta=2$ the distribution function (6) becomes

$F(x)=\left[1-e^{-\left(\theta x+\gamma x^{2}\right)}\right]^{\alpha}$

Transmuted generalized linear failure rate distribution. For $\beta=2$ the distribution function (6) becomes

$F(x)=\left[1-e^{-\left(\theta x+\gamma x^{2}\right)}\right]^{\alpha}\left((1+\lambda)-\lambda\left[1-e^{-\left(\theta x+\gamma x^{2}\right)}\right]^{\alpha}\right)$

Linear failure rate distribution. For $\lambda=0, \alpha=1, \beta=2$ the distribution function (6) becomes

$F(x)=1-e^{-\left(\theta x+\gamma x^{2}\right)}$

Transmuted linear failure rate distribution. For $\alpha=1, \beta=2$ the distribution function (6) becomes

$F(x)=\left(1-e^{-\left(\theta x+\gamma x^{2}\right)}\right)\left(1+\lambda e^{-\left(\theta x+\gamma x^{2}\right)}\right)$

Generalized Rayleigh distribution. For $\lambda, \theta=0, \beta=2$ the distribution function (6) becomes

$F(x)=\left[1-e^{-\left(\gamma x^{2}\right)}\right]^{\alpha}$

Transmuted generalized Rayleigh distribution. for $\theta=0, \beta=2$ the distribution function (6) becomes 
$F(x)=\left[1-e^{-\left(\gamma x^{2}\right)}\right]^{\alpha}\left((1+\lambda)-\lambda\left[1-e^{-\left(\gamma x^{2}\right)}\right]^{\alpha}\right)$

Rayleigh distribution. $\lambda, \theta=0, \alpha=1, \beta=2$ the distribution function (6) becomes

$F(x)=1-e^{-\left(\gamma x^{2}\right)}$

Transmuted Rayleigh distribution. $\theta=0, \alpha=1, \beta=2$ the distribution function (6) becomes

$F(x)=\left[1-e^{-\left(\gamma x^{2}\right)}\right]\left[1+\lambda e^{-\left(\gamma x^{2}\right)}\right]$

From the above, we see that the transmuted exponentiated modified Weibull distribution includes many of the popular lifetime distribution and represents therefore a considerable generalization. Figure 1 illustrates some of the possible shapes of the density function and distribution function of transmuted exponentiated modified Weibull distribution for selected values of the parameters.
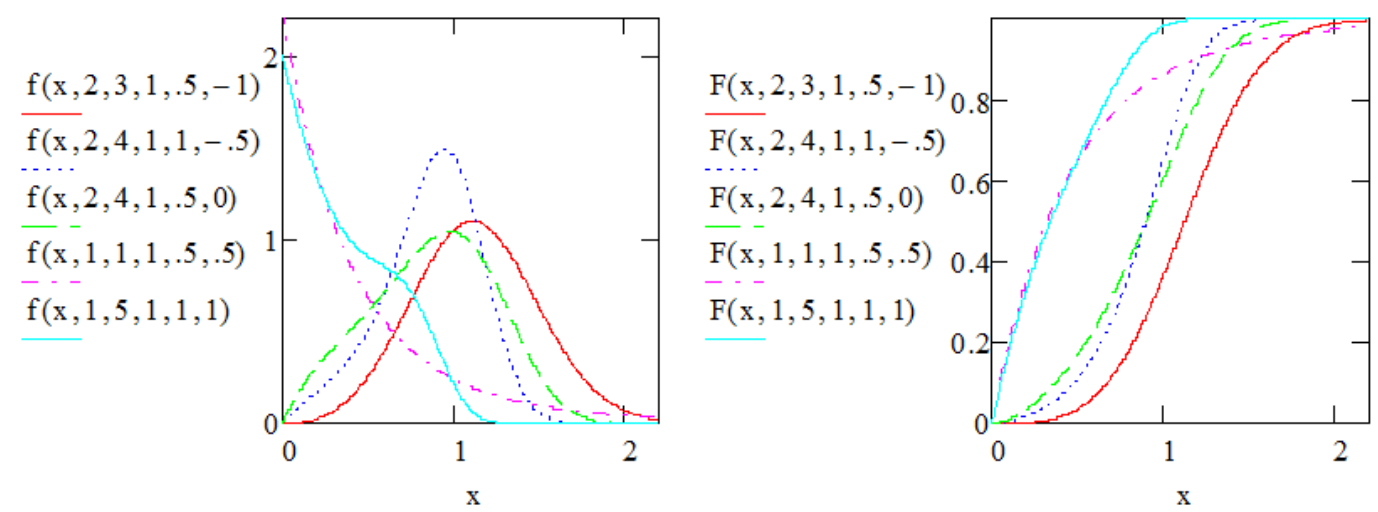

Fig. 1: Transmuted exponentiated modified Weibull Pdf\&CDF

\section{Reliability analysis}

The transmuted exponentiated modified Weibull distribution can be a useful characterization of life time data analysis. The reliability function (RF) of the transmuted exponentiated modified Weibull distribution is given by

$R(x)=1-\left[1-e^{-\left(\theta x+\gamma x^{\beta}\right)}\right]^{\alpha}\left((1+\lambda)-\lambda\left[1-e^{-\left(\theta x+\gamma x^{\beta}\right)}\right]^{\alpha}\right)$

It is important to note that $R(x)+F(x)=1$. One of the characteristic in reliability analysis is the hazard rate function defined by

$h(x)=\frac{f(x)}{1-F(x)}$

The hazard function of the transmuted exponentiated modified Weibull distribution also known as instantaneous failure rate denoted by $h(x)$ and is defined as $\frac{f(x)}{R(x)}$.

$h(x)=\frac{\alpha\left(\theta+\gamma \beta x^{\beta-1}\right) e^{-\left(\theta x+\gamma x^{\beta}\right)}\left[1-e^{-\left(\theta x+\gamma x^{\beta}\right)}\right]^{\alpha-1}\left(1+\lambda-2 \lambda\left[1-e^{-\left(\theta x+\gamma x^{\beta}\right)}\right]^{\alpha}\right)}{1-\left[1-e^{-\left(\theta x+\gamma x^{\beta}\right)}\right]^{\alpha}\left((1+\lambda)-\lambda\left[1-e^{-\left(\theta x+\gamma x^{\beta}\right)}\right]^{\alpha}\right)}$

It is important to note that the units for $h(x)$ is the probability of failure per unit of time, distance or cycles. Figure 2 shows the transmuted exponentiated modified Weibull instantaneous failure rate patterns. These failure rates are defined with different choices of parameters. 


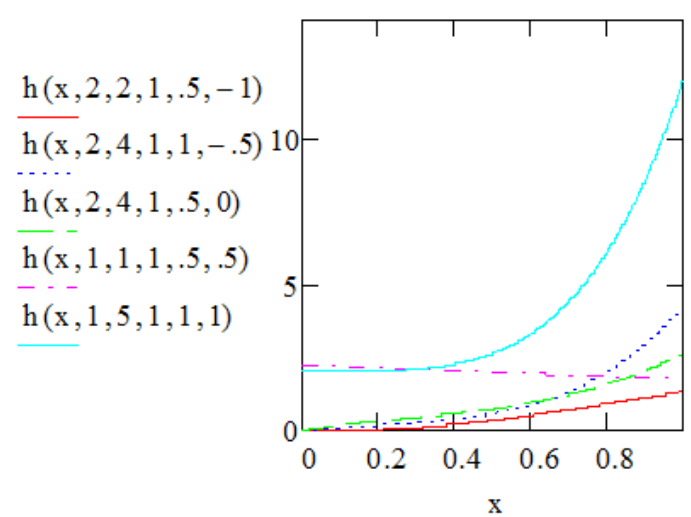

Fig 2: Transmuted exponentiated modified Weibull hazard rate function

Note that when $\alpha=1, \beta=5$ the distribution has the strictly increasing hazard rate function. when $\beta=1$, the hazard rate function is steadily increasing. when $\beta>1$, the hazard rate function is continually increasing. The hazard rate function of TEMWD as given in equation (28) becomes identical with the hazard rate function of transmuted exponentiated modified Rayleigh distribution for $\beta=2$ and for $\alpha, \beta=1$ it coincides with the transmuted modified exponential distribution. So the transmuted exponentiated modified Weibull is a very flexible reliability model.

Observing the behavior of the hazard rate function it is worth noting that the transmuted exponentiated modified Weibull distribution will have more applicability than the exponentiated modified Weibull distribution and some of its generalizations.

The hazard rate function of the transmuted exponentiated modified Weibull distribution has the following properties:

i. If $\lambda=0$ the failure rate is same as the exponentiated modified Weibull distribution

$$
h(x)=\frac{\alpha\left(\theta+\gamma \beta x^{\beta-1}\right) e^{-\left(\theta x+\gamma x^{\beta}\right)}\left[1-e^{-\left(\theta x+\gamma x^{\beta}\right)}\right]^{\alpha-1}}{1-\left[1-e^{-\left(\theta x+\gamma x^{\beta}\right)}\right]^{\alpha}}
$$

ii. If $\lambda=0, \alpha=1$ the failure rate is same as the modified Weibull distribution

$$
h(x)=\left(\theta+\gamma \beta x^{\beta-1}\right)
$$

iii. If $\alpha=1$ the failure rate is same as the transmuted modified Weibull distribution

$$
h(x)=\frac{\left(\theta+\gamma \beta x^{\beta-1}\right) e^{-\left(\theta x+\gamma x^{\beta}\right)}\left(1+\lambda-2 \lambda\left[1-e^{-\left(\theta x+\gamma x^{\beta}\right)}\right]\right)}{1-\left[1-e^{-\left(\theta x+\gamma x^{\beta}\right)}\right]\left(1+\lambda e^{-\left(\theta x+\gamma x^{\beta}\right)}\right)}
$$

iv. If $\lambda, \theta=0$ the failure rate is same as the exponentiated Weibull distribution

$$
h(x)=\frac{\alpha\left(\gamma \beta x^{\beta-1}\right) e^{-\left(\gamma x^{\beta}\right)}\left[1-e^{-\left(\gamma x^{\beta}\right)}\right]^{\alpha-1}}{1-\left[1-e^{-\left(\gamma x^{\beta}\right)}\right]^{\alpha}}
$$

v. If $\theta=0$ the failure rate is same as the transmuted exponentiated Weibull distribution

$$
h(x)=\frac{\alpha\left(\gamma \beta x^{\beta-1}\right) e^{-\left(\gamma x^{\beta}\right)}\left[1-e^{-\left(\gamma x^{\beta}\right)}\right]^{\alpha-1}\left(1+\lambda-2 \lambda\left[1-e^{-\left(\gamma x^{\beta}\right)}\right]^{\alpha}\right)}{1-\left[1-e^{-\left(\gamma x^{\beta}\right)}\right]^{\alpha}\left((1+\lambda)-\lambda\left[1-e^{-\left(\gamma x^{\beta}\right)}\right]^{\alpha}\right)}
$$

vi. If $\lambda, \theta=0$ and $\alpha=1$ the failure rate is same as the Weibull distribution

$$
h(x)=\gamma \beta x^{\beta-1}
$$

vii. If $\theta=0, \alpha=1$ the failure rate is same as the transmuted Weibull distribution

$$
h(x)=\frac{\left(\gamma \beta x^{\beta-1}\right) e^{-\left(\gamma x^{\beta}\right)}\left(1+\lambda-2 \lambda\left[1-e^{-\left(\gamma x^{\beta}\right)}\right]\right)}{1-\left[1-e^{-\left(\gamma x^{\beta}\right)}\right]\left(1+\lambda e^{-\left(\gamma x^{\beta}\right)}\right)}
$$

viii. If $\lambda, \theta=0$ and $\beta=1$ the failure rate is same as the exponentiated exponential distribution 


$$
h(x)=\frac{\alpha \gamma e^{-(\gamma x)}\left[1-e^{-(\gamma x)}\right]^{\alpha-1}}{1-\left[1-e^{-(\gamma x)}\right]^{\alpha}}
$$

ix. If $\theta=0$ and $\beta=1$ the failure rate is same as the transmuted exponentiated exponential distribution

$$
h(x)=\frac{\alpha \gamma e^{-(\gamma x)}\left[1-e^{-(\gamma x)}\right]^{\alpha-1}\left(1+\lambda-2 \lambda\left[1-e^{-(\gamma x)}\right]^{\alpha}\right)}{1-\left[1-e^{-(\gamma x)}\right]^{\alpha}\left((1+\lambda)-\lambda\left[1-e^{-(\gamma x)}\right]^{\alpha}\right)}
$$

x. If $\lambda, \theta=0$ and $\alpha, \beta=1$ the failure rate is same as the exponential distribution

$$
h(x)=\gamma
$$

xi. If $\theta=0$ and $\alpha, \beta=1$ the failure rate is same as the transmuted exponential distribution

$$
h(x)=\frac{\gamma e^{-(\gamma x)}\left(1+\lambda-2 \lambda\left[1-e^{-(\gamma x)}\right]\right)}{1-\left[1-e^{-(\gamma x)}\right]\left(1+\lambda e^{-(\gamma x)}\right)}
$$

xii. If $\lambda=0$ and $\beta=2$ the failure rate is same as the generalized linear failure rate distribution

$$
h(x)=\frac{\alpha(\theta+2 \gamma x) e^{-\left(\theta x+\gamma x^{2}\right)}\left[1-e^{-\left(\theta x+\gamma x^{2}\right)}\right]^{\alpha-1}}{1-\left[1-e^{-\left(\theta x+\gamma x^{2}\right)}\right]^{\alpha}}
$$

xiii. If $\beta=2$ the failure rate is same as the transmuted generalized linear failure rate distribution

$$
h(x)=\frac{\alpha(\theta+2 \gamma x) e^{-\left(\theta x+\gamma x^{2}\right)}\left[1-e^{-\left(\theta x+\gamma x^{2}\right)}\right]^{\alpha-1}\left(1+\lambda-2 \lambda\left[1-e^{-\left(\theta x+\gamma x^{2}\right)}\right]^{\alpha}\right)}{1-\left[1-e^{-\left(\theta x+\gamma x^{2}\right)}\right]^{\alpha}\left((1+\lambda)-\lambda\left[1-e^{-\left(\theta x+\gamma x^{2}\right)}\right]^{\alpha}\right)}
$$

xiv. If $\lambda=0, \alpha=1$ and $\beta=2$ the failure rate is same as the linear failure rate distribution

$$
h(x)=(\theta+2 \gamma x)
$$

xv. If $\alpha=1$ and $\beta=2$ the failure rate is same as the transmuted linear failure rate distribution

$$
h(x)=\frac{(\theta+2 \gamma x) e^{-\left(\theta x+\gamma x^{2}\right)}\left(1+\lambda-2 \lambda\left[1-e^{-\left(\theta x+\gamma x^{2}\right)}\right]\right)}{1-\left[1-e^{-\left(\theta x+\gamma x^{2}\right)}\right]\left(1+\lambda e^{-\left(\theta x+\gamma x^{2}\right)}\right)}
$$

xvi. If $\lambda, \theta=0$ and $\beta=2$ the failure rate is same as the generalized Rayleigh distribution

$$
h(x)=\frac{\alpha(2 \gamma x) e^{-\left(\gamma x^{2}\right)}\left[1-e^{-\left(\gamma x^{2}\right)}\right]^{\alpha-1}}{1-\left[1-e^{-\left(\gamma x^{2}\right)}\right]^{\alpha}}
$$

xvii. If $\theta=0$ and $\beta=2$ the failure rate is same as the transmuted generalized Rayleigh distribution

$$
h(x)=\frac{\alpha(2 \gamma x) e^{-\left(\gamma x^{2}\right)}\left[1-e^{-\left(\gamma x^{2}\right)}\right]^{\alpha-1}\left(1+\lambda-2 \lambda\left[1-e^{-\left(\gamma x^{2}\right)}\right]^{\alpha}\right)}{1-\left[1-e^{-\left(\gamma x^{2}\right)}\right]^{\alpha}\left((1+\lambda)-\lambda\left[1-e^{-\left(\gamma x^{2}\right)}\right]^{\alpha}\right)}
$$

xviii. If $\theta=0, \alpha=1$ and $\beta=2$ the failure rate is same as the transmuted Rayleigh distribution

$$
h(x)=\frac{(2 \gamma x) e^{-\left(\gamma x^{2}\right)}\left(1+\lambda-2 \lambda\left[1-e^{-\left(\gamma x^{2}\right)}\right]\right)}{1-\left[1-e^{-\left(\gamma x^{2}\right)}\right]\left(1+\lambda\left[1-e^{-\left(\gamma x^{2}\right)}\right]\right)}
$$

xix. If $\lambda, \theta=0, \alpha=1$ and $\beta=2$ the failure rate is same as the Rayleigh distribution

$$
h(x)=2 \gamma x
$$

Many generalized probability models have been proposed in reliability literature through the fundamental relationship between the reliability function $R(x)$ and its cumulative hazard function (CHF) $H(x)$ given by $-\ln R(x)$. The CHF describes how the risk of a particular outcome changes with time.

The cumulative hazard function of the transmuted exponentiated modified Weibull distribution is denoted by $H(x)$ and is defined as

Observe that:

$$
H(x)=-\ln \left(1-\left[1-e^{-\left(\theta x+\gamma x^{\beta}\right)}\right]^{\alpha}\left((1+\lambda)-\lambda\left[1-e^{-\left(\theta x+\gamma x^{\beta}\right)}\right]^{\alpha}\right)\right)
$$


i. $\quad H(t)$ is nondecreasing for all $t \geq 0$,

ii. $H(0)=0$,

iii. $\lim _{t \rightarrow \infty} H(t)=\infty$.

It is important to note that the units for $H(x)$ is the cumulative probability of failure per unit of time, distance or cycles. Figure 3 shows the transmuted exponentiated modified Weibull cumulative hazard function with different choices of parameters. For all choice of parameters the distribution has the non decreasing patterns of cumulative instantaneous hazard function.

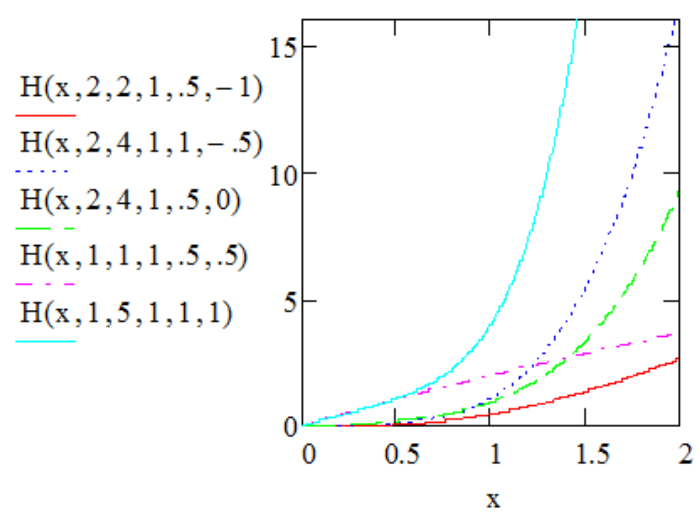

Fig 3: Transmuted exponentiated modified Weibull reliability cumulative hazard function

\section{Statistical properties}

This section explains the statistical properties of the TEMWD.

\subsection{Quantile and median}

The $q^{\text {th }}$ quantile $x_{q}$ of the transmuted exponentiated modified Weibull distribution is the real solution of the following equation

$\theta x_{q}+\gamma x_{q}^{\beta}+\ln \left(1-\left[\frac{(1+\lambda)-\sqrt{(1+\lambda)^{2}-4 \lambda q}}{2 \lambda}\right]^{1 / \alpha}\right)=0$

The equation (29) has no closed-form solution in $x_{q}$, so we have different cases by substituting the parametric values in the above quantile equation. So the derived cases are

(1) The q-th quantile of the Transmuted generalized linear failure rate distribution by substituting $\beta=2$

$x_{q}=\frac{-\theta+\sqrt{\theta^{2}-4 \gamma \ln \left(1-\left(\frac{(1+\lambda)-\sqrt{(1+\lambda)^{2}-4 \lambda q}}{2 \lambda}\right)^{1 / \alpha}\right)}}{2 \gamma}$

(2) The q-th quantile of the exponentiated Weibull by substituting $\theta=0$

$x_{q}=\left(-\frac{1}{\gamma} \ln \left(1-\left(\frac{(1+\lambda)-\sqrt{(1+\lambda)^{2}-4 \lambda q}}{2 \lambda}\right)^{1 / \alpha}\right)\right)^{1 / \beta}$

(3) The q-th quantile of the transmuted generalized Rayleigh distribution by substituting $\theta=0, \beta=2$ 
$x_{q}=\sqrt{-\frac{1}{\gamma} \ln \left(1-\left(\frac{(1+\lambda)-\sqrt{(1+\lambda)^{2}-4 \lambda q}}{2 \lambda}\right)^{1 / \alpha}\right)}$

(4) The q-th quantile of the transmuted exponentiated exponential distribution by substituting $\theta=0, \beta=1$

$x_{q}=-\frac{1}{\gamma} \ln \left(1-\left(\frac{(1+\lambda)-\sqrt{(1+\lambda)^{2}-4 \lambda q}}{2 \lambda}\right)^{1 / \alpha}\right)$

By putting $q=0.5$ in equation (29) we can get the median of TEMWD. The median life of the subject distribution is the 50 th percentile. In practice, this is the life by which 50 percent of the units will be expected to have failed and it is the life at which 50 percent of the units would be expected to still survive. To illustrate the effect of the shape parameter $\lambda$ on skewness and kurtosis we consider measures based on quantiles. The shortcomings of the classical kurtosis measure are well known. There are many heavy-tailed distributions for which this measure is infinite, so it becomes uninformative. The Bowley's skewness [6] is one of the earliest skewness measures defined by the average of the quartiles minus the median, divided by the half the interquartile range, given by

$\mathcal{B}=\frac{Q_{3}+Q_{1}-2 Q_{2}}{Q_{3}-Q_{1}}=\frac{Q(3 / 4)+Q(1 / 4)-2 Q(2 / 4)}{Q(3 / 4)-Q(1 / 4)}$.

and the Moors kurtosis [10] is based on octiles and is given by

$\mathcal{M}=\frac{\left(E_{3}-E_{1}\right)+\left(E_{7}-E_{5}\right)}{E_{6}-E_{2}}=\frac{Q(3 / 8)-Q(1 / 8)+Q(7 / 8)-Q(5 / 8)}{Q(6 / 8)-Q(2 / 8)}$.

For any distribution symmetrical to 0 the Moors kurtosis reduces to

$\mathcal{M}=\frac{\left(E_{7}-E_{5}\right)}{E_{6}}$.

It is easy to calculate that for standand normal distribution $E_{1}=-E_{7}=-1.15, E_{2}=-E_{6}=-0.32$. Therefore, $\mathcal{M}=1.23$. Hence, the centered Moor's coefficient is given by

$\mathcal{M}=\frac{\left(E_{3}-E_{1}\right)+\left(E_{7}-E_{5}\right)}{E_{6}-E_{2}}-1.23$

Fiqures 4 displays the Bowley $(\mathcal{B})$ and Moors $(\mathcal{M})$ kurtosis as a function of the parmeter $1 \geq \lambda>0$ and $-1 \leq \lambda<0$ for $\alpha, \beta=2, \theta=1$ and $\gamma=0.5$. It is evident that both measures depend on the parameter $\lambda$.
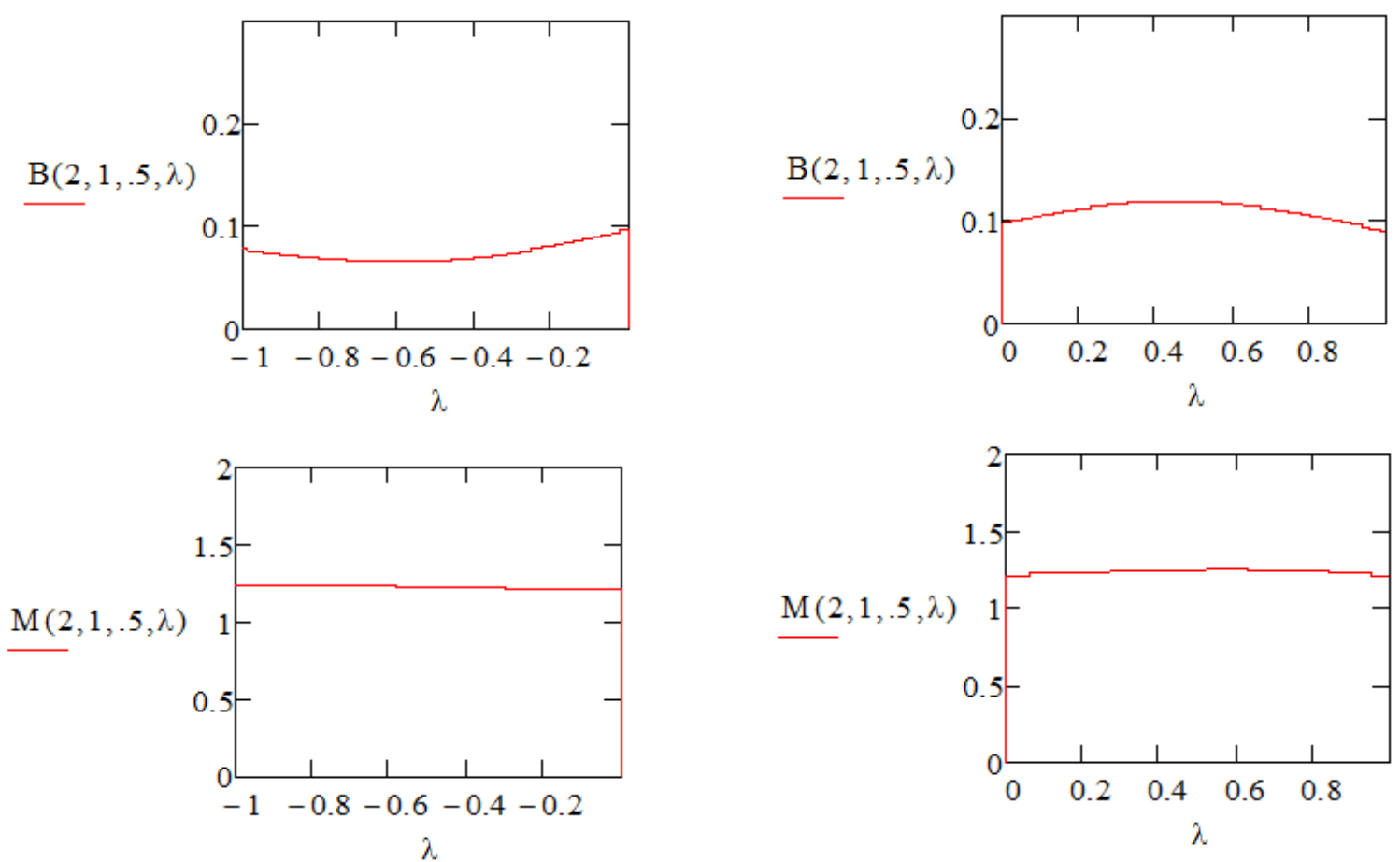

Fig 4: Behavior of Bowley(B) and Moors(M) kurtosis for TEMWD distribution. 


\subsection{Random number generation}

The random number as $x$ of the TEMWD is defined by the following equation

$\left[1-e^{-\left(\theta x+\gamma x^{\beta}\right)}\right]^{\alpha}\left((1+\lambda)-\lambda\left[1-e^{-\left(\theta x+\gamma x^{\beta}\right)}\right]^{\alpha}\right)=\xi$, where $\quad \xi \sim U(0,1)$

$\theta x+\gamma x^{\beta}+\ln \left(1-\left[\frac{(1+\lambda)-\sqrt{(1+\lambda)^{2}-4 \lambda \xi}}{2 \lambda}\right]^{1 / \alpha}\right)=0$

The above equation is not in closed form solution in $x$, using $\xi$ a random number uniformly distributed from zero to one, we have solved the above equation $F(x)=\xi$ to obtain a random number in $x$.

\subsection{Moments}

The $r^{\text {th }}$ moments of a transmuted exponentiated modified Weibull random variable $X$, is given by

$$
\begin{gathered}
E\left(X^{r}\right)=\int_{0}^{\infty} \alpha X^{r}\left(\theta+\gamma \beta x^{\beta-1}\right) e^{-\left(\theta x+\gamma x^{\beta}\right)}\left[1-e^{-\left(\theta x+\gamma x^{\beta}\right)}\right]^{\alpha-1}\left(1+\lambda-2 \lambda\left[1-e^{-\left(\theta x+\gamma x^{\beta}\right)}\right]^{\alpha}\right), \\
=\alpha \sum_{j=0}^{\infty} \sum_{k=0}^{\infty}\left[(1+\lambda)\left(\begin{array}{c}
\alpha-1 \\
k
\end{array}\right)-2 \lambda\left(\begin{array}{c}
2 \alpha-1 \\
k
\end{array}\right)\right] \frac{(-1)^{j+k}(\gamma(k+1))^{j}}{j !} \\
\times\left(\frac{\theta \Gamma(r+\beta j+1)}{(\theta(k+1))^{r+\beta j+1}}+\beta \gamma \frac{\Gamma(r+\beta j+\beta)}{(\theta(k+1))^{r+\beta j+\beta}}\right)
\end{gathered}
$$

Based on the equation (31), the coefficient of variation, coefficient of skewness and coefficient of kurtosis of TEMWD can be obtained according to the following relation

$$
\begin{aligned}
& C V=\sqrt{\frac{\mu_{2}}{\mu_{1}^{2}}-1} \\
& C S=\frac{\mu_{3}-3 \mu_{2} \mu_{1}+2 \mu_{1}^{3}}{\left(\mu_{2}-\mu_{1}^{2}\right)^{\frac{3}{2}}} \\
& C K=\frac{\mu_{4}-4 \mu_{3} \mu_{1}+6 \mu_{2} \mu_{1}^{2}-3 \mu_{1}^{4}}{\left(\mu_{2}-\mu_{1}^{2}\right)^{2}}
\end{aligned}
$$

The moment generating function of the TEMWD is given by

$M(t)=\alpha \sum_{j=0}^{\infty} \sum_{k=0}^{\infty}\left[(1+\lambda)\left(\begin{array}{c}\alpha-1 \\ k\end{array}\right)-2 \lambda\left(\begin{array}{c}2 \alpha-1 \\ k\end{array}\right)\right] \frac{(-1)^{j+k}(\gamma(k+1))^{j}}{j !} \times\left(\frac{\theta \Gamma(\beta k+1)}{(\theta(j+1)-t)^{\beta k+1}}+\beta \gamma \frac{\Gamma(\beta k+\beta)}{(\theta(j+1)-t)^{\beta k+\beta}}\right)$

Based on the equation (35), the measure of central tendency, measure of dispersion, coefficient of variation, coefficient of skewness and coefficient of kurtosis of TEMWD can be obtained.

\section{Least square estimation}

Let $X_{1}, X_{2}, \ldots X_{n}$ be a sample size $\mathrm{n}$ from a transmuted exponentiated modified Weibull distribution with cdf $F(x)$, and suppose that $X_{(i)}, i=1,2, \ldots, n$ denote the ordered sample. For sample of size $n$, we have

$E\left(F\left(T_{(i)}\right)\right)=\frac{i}{n+1}$

The least square estimators (LSES) are obtained by minimizing

$Q(\alpha, \beta, \theta, \gamma, \lambda)=\sum_{i=0}^{n}\left(F\left(T_{(i)}\right)-\frac{i}{n+1}\right)^{2}$

By using (3) and (36) we have the following equation 
$Q(\alpha, \beta, \theta, \gamma, \lambda)=\sum_{i=0}^{n}\left(\left[1-e^{-\left(\theta x+\gamma x^{\beta}\right)}\right]^{\alpha}\left((1+\lambda)-\lambda\left[1-e^{-\left(\theta x+\gamma x^{\beta}\right)}\right]^{\alpha}\right)-\frac{i}{n+1}\right)^{2}$

To minimize equation (37) with respect to $\alpha, \beta, \theta, \gamma$ and $\lambda$, we differentiate with respect to these parameters, which leads to the following equations

$$
\begin{aligned}
& \frac{d Q}{d \alpha}=\sum_{i=0}^{n}\left(\left[1-e^{-\left(\theta x+\gamma x^{\beta}\right)}\right]^{\alpha}\left((1+\lambda)-\lambda\left[1-e^{-\left(\theta x+\gamma x^{\beta}\right)}\right]^{\alpha}\right)-\frac{i}{n+1}\right) \\
& \times\left[1-e^{-\left(\theta x+\gamma x^{\beta}\right)}\right]^{\alpha} \ln \left(1-e^{-\left(\theta x+\gamma x^{\beta}\right)}\right)\left((1+\lambda)-2 \lambda\left[1-e^{-\left(\theta x+\gamma x^{\beta}\right)}\right]^{\alpha}\right), \\
& \frac{d Q}{d \beta}=\left(\left[1-e^{-\left(\theta x+\gamma x^{\beta}\right)}\right]^{\alpha}\left((1+\lambda)-\lambda\left[1-e^{-\left(\theta x+\gamma x^{\beta}\right)}\right]^{\alpha}\right)-\frac{i}{n+1}\right) \\
& \times\left(\gamma \alpha x^{\beta} e^{-\left(\theta x+\gamma x^{\beta}\right)} \ln x\left[1-e^{-\left(\theta x+\gamma x^{\beta}\right)}\right]^{\alpha-1}\right)\left((1+\lambda)\left(1-\left[1-e^{-\left(\theta x+\gamma x^{\beta}\right)}\right]^{\alpha}\right)\right), \\
& \frac{d Q}{d \theta}=\left(\left[1-e^{-\left(\theta x+\gamma x^{\beta}\right)}\right]^{\alpha}\left((1+\lambda)-\lambda\left[1-e^{-\left(\theta x+\gamma x^{\beta}\right)}\right]^{\alpha}\right)-\frac{i}{n+1}\right) \\
& \times\left(\alpha x e^{-\left(\theta x+\gamma x^{\beta}\right)}\left[1-e^{-\left(\theta x+\gamma x^{\beta}\right)}\right]^{\alpha-1}\left((1+\lambda)-2 \lambda\left[1-e^{-\left(\theta x+\gamma x^{\beta}\right)}\right]^{\alpha}\right)\right), \\
& \frac{d Q}{d \gamma}=\left(\left[1-e^{-\left(\theta x+\gamma x^{\beta}\right)}\right]^{\alpha}\left((1+\lambda)-\lambda\left[1-e^{-\left(\theta x+\gamma x^{\beta}\right)}\right]^{\alpha}\right)-\frac{i}{n+1}\right) \\
& \times\left(\alpha x^{\beta} e^{-\left(\theta x+\gamma x^{\beta}\right)}\left[1-e^{-\left(\theta x+\gamma x^{\beta}\right)}\right]^{\alpha-1}\left((1+\lambda)-2 \lambda\left[1-e^{-\left(\theta x+\gamma x^{\beta}\right)}\right]^{\alpha}\right)\right) \\
& \frac{d Q}{d \lambda}=\left(\left[1-e^{-\left(\theta x+\gamma x^{\beta}\right)}\right]^{\alpha}\left((1+\lambda)-\lambda\left[1-e^{-\left(\theta x+\gamma x^{\beta}\right)}\right]^{\alpha}\right)-\frac{i}{n+1}\right) \\
& \times\left(\left[1-e^{-\left(\theta x+\gamma x^{\beta}\right)}\right]^{\alpha}-1\right)\left[1-e^{-\left(\theta x+\gamma x^{\beta}\right)}\right]^{\alpha} .
\end{aligned}
$$

The estimates of the parameters $\alpha, \beta, \theta, \gamma$ and $\lambda$ are obtained by equating the above equations to zero. Although the proposed estimators cannot be expressed in closed form, they can be obtained through the use of an appropriate numerical solution algorithm.

\section{Order statistics}

Order statistics make their appearance in many areas of statistical theory and practice. We know that if $X_{(1)}, X_{(2)}, \ldots, X_{(n)}$ denotes the order statistics of a random sample $X_{1}, X_{2}, \ldots, X_{n}$ from a continuous population with cdf $F_{X}(x)$ and pdf $f_{X}(x)$, then the pdf of $X_{(j)}$ is given by

$f_{X_{(j)}}(x)=\frac{n !}{(j-1) !(n-j) !} f_{X}(x)\left[F_{X}(x)\right]^{j-1}\left[1-F_{X}(x)\right]^{n-j}$

For $j=1,2, \ldots, n$

We have from (4) and (5) the pdf of the $j^{\text {th }}$ order exponentiated modified Weibull random variable $X_{(j)}$ given by $\mathrm{g}_{X_{(j)}}(x)=\frac{n ! \alpha\left(\theta+\gamma \beta x^{\beta-1}\right) e^{-\left(\theta x+\gamma x^{\beta}\right)}\left[1-e^{-\left(\theta x+\gamma x^{\beta}\right)}\right]^{\alpha j-1}}{(j-1) !(n-j) !}\left[1-\left[1-e^{-\left(\theta x+\gamma x^{\beta}\right)}\right]^{\alpha}\right]^{n-j}$

therefore, the pdf of the $n^{\text {th }}$ order exponentiated modified Weibull statistic $X_{(n)}$ is given by 
$\mathrm{g}_{X_{(n)}}(x)=n \alpha\left(\theta+\gamma \beta x^{\beta-1}\right) e^{-\left(\theta x+\gamma x^{\beta}\right)}\left[1-e^{-\left(\theta x+\gamma x^{\beta}\right)}\right]^{\alpha n-1}$

and the pdf of the first order exponentiated modified Weibull statistic $X_{(1)}$ is given

$\mathrm{g}_{X_{(1)}}(x)=n \alpha\left(\theta+\gamma \beta x^{\beta-1}\right) e^{-\left(\theta x+\gamma x^{\beta}\right)}\left[1-e^{-\left(\theta x+\gamma x^{\beta}\right)}\right]^{\alpha-1}\left[1-\left[1-e^{-\left(\theta x+\gamma x^{\beta}\right)}\right]^{\alpha}\right]^{n-1}$

Note that in a particular case of $n=2,(38)$ yields

$\mathrm{g}_{X_{(2)}}(x)=2 \alpha\left(\theta+\gamma \beta x^{\beta-1}\right) e^{-\left(\theta x+\gamma x^{\beta}\right)}\left[1-e^{-\left(\theta x+\gamma x^{\beta}\right)}\right]^{2 \alpha-1}$

and (39) yields

$\mathrm{g}_{X_{(1)}}(x)=2 \alpha\left(\theta+\gamma \beta x^{\beta-1}\right) e^{-\left(\theta x+\gamma x^{\beta}\right)}\left[1-e^{-\left(\theta x+\gamma x^{\beta}\right)}\right]^{\alpha-1} \times\left[1-\left[1-e^{-\left(\theta x+\gamma x^{\beta}\right)}\right]^{\alpha}\right]$

Observe that (40) and (41) are special cases of (7) for $\lambda=-1$ and $\lambda=1$ respectively. It has been observed that a transmuted exponentiated modified Weibull distribution with $\lambda=1$ is the distribution of $\min \left(X_{1}, X_{2}\right)$ and a transmuted exponentiated Modified Weibull with $\lambda=-1$ is the of the $\max \left(X_{1}, X_{2}\right)$ where $X_{1}$ and $X_{2}$ are independent and identically distributed exponentiated modified Weibull random variables.

Now we provide the distribution of the order statistics for transmuted exponentiated modified Weibull random variable. The pdf of the $j^{t h}$ order statistic for transmuted exponetiated Modified Weibull distribution is given by

$$
\begin{aligned}
\left.f_{X_{(j)}}(x)=\frac{n ! \alpha(\theta}{}+\gamma \beta x^{\beta-1}\right) e^{-\left(\theta x+\gamma x^{\beta}\right)} & (j-1) !(n-j) ! \\
& \times\left[\left((1+\lambda)-\lambda\left[1-e^{-\left(\theta x+\gamma x^{\beta}\right)}\right]^{\alpha j-1}\left(\left[1+\lambda-2 \lambda\left[1-e^{-\left(\theta x+\gamma x^{\beta}\right)}\right]^{\alpha}\right]\right.\right.\right. \\
& \left.\left.\times\left[1-\left[1-e^{-\left(\theta x+\gamma x^{\beta}\right)}\right]^{\alpha}((1+\lambda)]^{j-1}-\lambda\left[1-e^{-\left(\theta x+\gamma x^{\beta}\right)}\right]^{\alpha}\right)\right]^{n-j}\right) .
\end{aligned}
$$

therefore, the pdf of the largest order statistic $X_{(n)}$ is given by

$$
\begin{aligned}
f_{X_{(n)}}(x)=n \alpha(\theta & \left.+\gamma \beta x^{\beta-1}\right) e^{-\left(\theta x+\gamma x^{\beta}\right)}\left[1-e^{-\left(\theta x+\gamma x^{\beta}\right)}\right]^{\alpha n-1}\left(\left[1+\lambda-2 \lambda\left[1-e^{-\left(\theta x+\gamma x^{\beta}\right)}\right]^{\alpha}\right]\right. \\
& \left.\times\left[\left((1+\lambda)-\lambda\left[1-e^{-\left(\theta x+\gamma x^{\beta}\right)}\right]^{\alpha}\right)\right]^{n-1}\right) .
\end{aligned}
$$

and the pdf of the smallest order statistic $X_{(1)}$ is given by

$$
\begin{aligned}
f_{X_{(1)}}(x)=n \alpha(\theta & \left.+\gamma \beta x^{\beta-1}\right) e^{-\left(\theta x+\gamma x^{\beta}\right)}\left[1-e^{-\left(\theta x+\gamma x^{\beta}\right)}\right]^{\alpha-1}\left(\left[1+\lambda-2 \lambda\left[1-e^{-\left(\theta x+\gamma x^{\beta}\right)}\right]^{\alpha}\right]\right. \\
& \left.\times\left[1-\left[1-e^{-\left(\theta x+\gamma x^{\beta}\right)}\right]^{\alpha}\left((1+\lambda)-\lambda\left[1-e^{-\left(\theta x+\gamma x^{\beta}\right)}\right]^{\alpha}\right)\right]^{n-1}\right) .
\end{aligned}
$$

Note that $\lambda=0$ yields the order statistics of the exponentiated modified Weibull distribution.

\section{Conclusion}

In the present study, we have introduced a new generalization of exponentiated modified Weibull distribution called the transmuted exponentiated modified Weibull distribution. The subject distribution is generated by using the quadratic rank transmutation map and taking the exponentiated modified Weibull distribution as the base distribution. Some mathematical properties along with estimation issues are addressed. The hazard rate function and reliability behavior of the transmuted exponentiated modified Weibull distribution shows that the subject distribution can be used to model 
reliability data. We expect that this study will serve as a reference and help to advance future research in the subject area.

\section{References}

[1] G. R. Aryal and C. P. Tsokos. On the transmuted extreme value distribution with application. Nonlinear Analysis: Theory, Methodsand Applications, 71, (2009) 1401-1407.

[2] G. R. Aryal and C. P. Tsokos. Transmuted Weibull distribution: A generalization of the Weibull probability distribution. European Journal of Pure and Applied Mathematics, 4(2), (2011) 89-102.

[3] G. R. Aryal. Transmuted Log-Logistic Distribution. Journal of Statistics Applications \& Probability. No. 1, (2013) 11-20.

[4] I. Elbatal. Exponentiated modified Weibull distribution. Economic Quality Control. 26, (2011) 189-200.

[5] R.D. Gupta and D. Kundu. Exponentiated exponential distribution. An alternative to gamma and Weibull distributions. Biometrical Journal 43(2001), 117-330.

[6] J. F. Kenney and E. S. Keeping. Mathematics of Statistics. Princeton, NJ, 1962.

[7] M. S. Khan and R. King. Transmuted modified Weibull Distribution: A generalization of the modified Weibull probability distribution. European Journal of Pure and Applied Mathematics. Vol. 6, No. 1, (2013), 66-88.

[8] D. Kundu and M. Z. Rakab. Generalized Rayleigh distribution: Different methods of estimation. Computational Statistics and Data Analysis 49 (2005), 187-200.

[9] C. D. Lai, M. Xie and D. N. P. Murthy. A modified Weibull distribution. IEEE Transaction on Reliability 52 (2003), 33-37.

[10] J. A. Moors. A quantile alternative for kurtosis. Journal of the Royal Statistical Society. D, 37, (1998).25-32.

[11] G. S. Mudholkar, D.K. Srivastava and M. Friemer. The exponentiated Weibull family: A reanalysis of the bus-motor-failure data Technometrics 37 (1995), 436-445.

[12] G. S. Mudholkar, D.K. Srivastava and G.D. Kollia. A generalization of the Weibull distribution with application to the analysis of survival data. Journal of the American Statistical Association 91 (1996), 1575-1583.

[13] W. Shaw and I. Buckley. The alchemy of probability distributions: beyond Gram- Charlier expansions, and a skew-kurtotic-normal distribution from a rank transmutation map. Research report, (2007). 